\title{
Is cardiac diagnosis a predictor of neurodevelopmental outcome after cardiac surgery in infancy?
}

\author{
J. William Gaynor, MD, ${ }^{\mathrm{a}}$ Marsha Gerdes, PhD, ${ }^{\mathrm{b}}$ Alex S. Nord, BA, ${ }^{\mathrm{c}}$ Judy Bernbaum, MD, ${ }^{\mathrm{d}}$ \\ Elaine Zackai, MD, ${ }^{\mathrm{e}}$ Gil Wernovsky, MD, ${ }^{\mathrm{f}}$ Robert R. Clancy, MD, ${ }^{\mathrm{g}}$ Patrick J. Heagerty, PhD, \\ Cynthia B. Solot, MA, CCC, ${ }^{\mathrm{i}}$ Donna McDonald-McGinn, MS, CGC, ${ }^{\mathrm{e}}$ and Gail P. Jarvik, MD, PhD
}

Objectives: To determine whether a cardiac diagnosis is a predictor of neurodevelopmental outcomes after infant cardiac surgery.

Methods: Infants with ventricular septal defect (VSD), tetralogy of Fallot (TOF), transposition of the great arteries (TGA), and hypoplastic left heart syndrome (HLHS) in a study of apolipoprotein E (APOE) polymorphisms, and neurodevelopmental outcome underwent neurodevelopmental and genetic evaluation at 4 years of age. The domains tested included cognition, language, speech, memory, executive function, visual-motor, fine motor, and reading and math skills.

Results: Testing was completed in 178 patients with normal genetic evaluations: VSD $(n=26)$, TOF $(n=44)$, TGA $(\mathrm{n}=41)$, and HLHS $(\mathrm{n}=67)$. No differences were found in gestational age, ethnicity, APOE genotype, socioeconomic status, or maternal education among groups. Patient age at the first surgery was significantly lower for patients with TGA and HLHS compared with those with TOF and VSD. The postoperative length of stay was significantly longer for HLHS than all other groups and for TGA compared with TOF and VSD. HLHS correlated significantly with the use of deep hypothermic circulatory arrest and multiple operations. The mean scores for each domain were within normal limits for all groups. Compared with the other patients, those with HLHS had significantly lower scores for cognition, fine motor skills, executive function, and math skills. No significant differences were found among the TGA, TOF or VSD patients for any domain. Significant impairments in at least 1 domain were identified in $8 \%(2 / 25)$ of patients with VSD, 20\% (8/41) with TOF, 17\% (7/41) with TGA, and 18\% (12/65) with HLHS. After correction for the demographic, preoperative, and operative variables, no significant differences were found among the groups for any domain.

Conclusions: The mean scores for the neurodevelopmental outcomes domains tested were in the normal range for preschool children with no recognized genetic syndromes after surgery for VSD, TOF, TGA, and HLHS. In each diagnostic group, the number of children with impairments in at least 1 domain increased compared with the general population. Unadjusted neurodevelopmental outcomes for HLHS were lower for cognition, fine motor skills, executive function, and math skills compared with the other patients. After correction for the demographic, preoperative, and operative variables, no significant differences were found among the groups for any domain. The specific cardiac diagnosis determines a large portion of the variation in these covariates. Therefore, although HLHS did predict for poorer outcomes in some domains, it did not add predictive power to the other factors considered. (J Thorac Cardiovasc Surg 2010;140:1230-7)

Congenital heart defects (CHDs) are the most common birth defects in humans, affecting 8/1000 live births ( 230 -

\footnotetext{
From the Division of Cardiothoracic Surgery, ${ }^{\mathrm{a}}$ the Division of Psychology, ${ }^{\mathrm{b}}$ the Division of General Pediatrics, ${ }^{\mathrm{d}}$ the Division of Genetics, ${ }^{\mathrm{e}}$ the Division of Pediatric Cardiology, ${ }^{\mathrm{f}}$ the Division of Neurology, ${ }^{\mathrm{g}}$ and the Center for Childhood Communication, ${ }^{\mathrm{i}}$ Children's Hospital of Philadelphia, Philadelphia, Pa; and the Department of Medicine (Medical Genetics), ${ }^{\mathrm{c}}$ and the Department of Biostatistics, ${ }^{\mathrm{h}}$ University of Washington, Seattle, Wash.

Disclosures: None.

Read at the 90th Annual Meeting of The American Association for Thoracic Surgery, Toronto, Ontario, Canada, May 1-5, 2010.

Received for publication April 27, 2010; revisions received July 23, 2010; accepted for publication July 30, 2010; available ahead of print Oct 18, 2010.

Address for reprints: J. William Gaynor, MD, Division of Cardiothoracic Surgery, Children's Hospital of Philadelphia, 34th and Civic Center Boulevard, Philadelphia, PA 19104 (E-mail: gaynor@email.chop.edu).

$0022-5223 / \$ 36.00$

Copyright (C) 2010 by The American Association for Thoracic Surgery doi: $10.1016 /$ j.jtcvs.2010.07.069
}

40,000 children annually in the United States) with one third of the affected children requiring intervention in early infancy. Improved survival, combined with the expectations for independence and behavioral self-regulation as the children mature, has led to a growing recognition of neurobehavioral symptoms and impaired functional outcomes in some survivors. Neurodevelopmental dysfunction is the most common, and potentially most disabling, outcome of CHDs and their treatment. ${ }^{1,2}$

The prevalence of adverse neurodevelopmental outcomes appears to vary according to the cardiac diagnosis. Previous studies have suggested that infants with transposition of the great arteries (TGA) undergoing the arterial switch tend to have better short- and intermediate-term neurodevelopmental outcomes compared with infants with other types of CHD suitable for 2-ventricle repair, such as 


$$
\begin{aligned}
& \text { Abbreviations and Acronyms } \\
& \begin{aligned}
\text { APOE } & =\text { apolipoprotein E } \\
\text { CPB } & =\text { cardiopulmonary bypass } \\
\text { CHD } & =\text { congenital heart defect } \\
\text { DHCA } & =\text { deep hypothermic circulatory arrest } \\
\text { GFTA } & =\text { Goldman Fristoe Test of Articulation } 2 \\
\text { HLHS } & =\text { hypoplastic left heart syndrome } \\
\text { ND } & =\text { neurodevelopmental } \\
\text { NEPSY } & =\text { Neuro-PSYchology Statue test } \\
\text { TGA } & =\text { transposition of great arteries } \\
\text { TOF } & =\text { tetralogy of Fallot } \\
\text { VMI } & =\text { visual motor integration } \\
\text { VSD } & =\text { ventricular septal defect }
\end{aligned}
\end{aligned}
$$

tetralogy of Fallot (TOF) and ventricular septal defect (VSD). ${ }^{3}$ Many studies have suggested that infants with functional single ventricle, especially hypoplastic left heart syndrome (HLHS), have a greater prevalence of adverse neurodevelopmental outcomes compared infants with other types of CHD. ${ }^{4,5}$ However, it is unclear whether these differences in outcomes result from differences in central nervous system development related to the type of CHD, differences in the physiologic effects of the specific defect, or diagnosis-specific differences in management.

Apolipoprotein E (APOE) is an important regulator of cholesterol metabolism and has an important role as a susceptibility gene that modifies the outcome after central nervous system injury. In 1998, we initiated a prospective study evaluating the association between neurodevelopmental dysfunction and the $A P O E$ genotype in 550 neonates and infants undergoing surgery for $\mathrm{CHD} .{ }^{6}$ The $A P O E \varepsilon 2$ allele was associated with a significantly worse neurodevelopmental outcome, including cognitive function and motor skills, at 1 year of age. The cohort subsequently underwent a detailed neurobehavioral evaluation at 4 to 5 years of age to test the hypothesis that the APOE genotype predicts the susceptibility for long-term neurodevelopmental dysfunction after cardiac surgery in infants. The risk of behavioral problems was modified by the $A P O E$ genotype. The $A P O E$ $\varepsilon 2$ allele was associated with increased behavior problems, impaired social interactions, and restricted behavior patterns. ${ }^{7}$ An additional aim of the evaluation was to evaluate the significance of cardiac diagnosis as an independent risk factor for adverse neurodevelopmental outcomes.

\section{MATERIALS AND METHODS}

The present study was a prospective trial evaluating the association between neurodevelopmental dysfunction and cardiac diagnosis in preschool patients (4-5 years of age) after cardiac surgery in infancy. ${ }^{6,7}$ Patients 6 months of age or younger undergoing surgery for CHD using cardiopulmonary bypass (CPB) with or without deep hypothermic circulatory arrest (DHCA) were eligible. The exclusion criteria included multiple congenital anomalies, a recognizable genetic or phenotypic syndrome other than chromosome 22q11 microdeletion syndrome, and language other than English spoken in the home. The Institutional Review Board at The Children's Hospital of Philadelphia approved the study, and the parent or guardian provided informed consent.

The population for the present study consisted of patients with TGA, TOF, VSD, or HLHS who returned for the evaluation and had normal genetic evaluation findings.

\section{Operative Management}

Alpha-stat blood gas management was used. The pump flow rates were not standardized for the present study. DHCA was used at the surgeon's discretion. Before DHCA, patients underwent core cooling, with topical hypothermia of the head to a nasopharyngeal temperature of $18^{\circ} \mathrm{C}$. Modified ultrafiltration was performed in all patients.

\section{Data Collection}

Preoperative factors that might independently affect the neurobehavioral outcomes, including gestational age, birth head circumference, and birth weight, were obtained from the hospital records. The weight and age at surgery were recorded for the initial surgery and subsequent procedures with $\mathrm{CPB}$. The operative variables were recorded, including the duration of $\mathrm{CPB}$ and DHCA, lowest nasopharyngeal temperature, and hematocrit after hemodilution.

\section{Four-Year Neurodevelopmental Examination}

The neurodevelopmental evaluation was performed between the fourth and fifth birthdays. The growth measurements (ie, weight, length, and head circumference) were recorded. The maternal education, Hollingshead socioeconomic status, and ethnicity were determined by parental report. A health history was obtained focusing on the incidence of interim illness: rehospitalizations, neurologic events or interim evaluations, current medications, and parental concerns about health.

Patients were evaluated by a genetic dysmorphologist at the 1 and/or 4 year evaluations. Additional genetic analyses were performed if indicated Neonatal recognition of the dysmorphic features can be difficult; therefore, some patients were enrolled for whom the diagnosis of a genetic syndrome was not made until a later evaluation. Patients were classified as having no definite genetic syndrome or chromosomal abnormality (normal), suspected genetic syndrome (suspect), or definite genetic or chromosomal abnormality (abnormal). Only patients classified as normal were included in the present analysis.

\section{APOE Genotype Determination}

Whole blood or a buccal swab was obtained before the operation and stored at $4^{\circ} \mathrm{C}$. Genomic DNA was prepared and used to determine the $A P O E$ genotypes using a previously published method. ${ }^{6}$

\section{Neurodevelopmental Testing}

To provide a broad assessment of neurodevelopmental status, multiple domains were tested, including cognition, language, speech, memory, executive function, and visual-motor, fine motor, and academic skills. Cognitive skills were assessed using the full-scale IQ from the Wechsler Preschool and Primary Scale of Intelligence, 3rd edition (WPPSI-III). ${ }^{8}$ Core language skill was assessed using the Preschool Language Scale-4 Total Language Score (PLS-4 TLS). ${ }^{9}$ Executive function was assessed using the Neuro-PSYchology statue test, which targets inhibition and motor persistence (component processes of attention and executive function). ${ }^{10}$ Visual-motor integration was assessed with the developmental test of Visual Motor Integration, a simple copying task that assesses the child's fine motor and visual motor coordination skills. ${ }^{11}$ Speech production was assessed using the Goldman Fristoe Test of Articulation 2 (GFTA). ${ }^{12}$ Academic achievement (school readiness for reading and math) was tested 
TABLE 1. Comparison of baseline and operative characteristics by diagnosis

\begin{tabular}{|c|c|c|c|c|c|}
\hline Baseline characteristic & TGA $(n=41)$ & TOF $(n=44)$ & $\operatorname{VSD}(\mathbf{n}=\mathbf{2 6})$ & HLHS $(n=67)$ & $P$ value \\
\hline \multicolumn{6}{|l|}{ Gender } \\
\hline Female & 15 & 23 & 10 & 27 & \\
\hline Male & 26 & 21 & 16 & 40 & \\
\hline \multicolumn{6}{|l|}{ Ethnicity } \\
\hline Asian-Pacific, Hispanic, Native American, or other & 8 & 7 & 2 & 5 & \\
\hline Non-Hispanic black & 5 & 8 & 5 & 13 & \\
\hline Non-Hispanic white & 28 & 29 & 19 & 49 & \\
\hline Gestational age (wk) & $\mathrm{n}=41$ & $\mathrm{n}=44$ & $\mathrm{n}=26$ & $\mathrm{n}=67$ & \\
\hline Mean \pm SD (n) & $39.1 \pm 1.6$ & $35.5 \pm 1.9$ & $38.6 \pm 2.0$ & $38.6 \pm 1.6$ & \\
\hline Birth weight $(\mathrm{g})$ & $\mathrm{n}=41$ & $\mathrm{n}=44$ & $\mathrm{n}=26$ & $\mathrm{n}=67$ & $.049 *$ \\
\hline Mean \pm SD (n) & $3284 \pm 486$ & $3055 \pm 568$ & $3225 \pm 678$ & $3275 \pm 593$ & \\
\hline Birth head circumference $(\mathrm{cm})$ & $\mathrm{n}=41$ & $\mathrm{n}=42$ & $\mathrm{n}=25$ & $\mathrm{n}=67$ & \\
\hline Mean \pm SD & $33.8 \pm 1.4$ & $33.6 \pm 2.0$ & $33.4 \pm 1.9$ & $34.1 \pm 1.9$ & \\
\hline Preoperative intubation (n) & $20(49 \%)$ & $1(2 \%)$ & $0(0 \%)$ & $29(43 \%)$ & $<.001 \dagger$ \\
\hline \multicolumn{6}{|l|}{ Operative characteristics (first procedure) } \\
\hline Age (d) & $4.7 \pm 5.4$ & $90.7 \pm 50.8$ & $102 \pm 37$ & $4.7 \pm 4.7$ & $<.001 \dagger$ \\
\hline Weight (kg) & $3.4 \pm 0.5$ & $5.1 \pm 1.4$ & $4.8 \pm 1.1$ & $3.3 \pm 0.6$ & $<.001 \dagger$ \\
\hline Total CPB time (min) & $114.3 \pm 55.5$ & $59.5 \pm 21.1$ & $45.8 \pm 16.3$ & $55.4 \pm 27.8$ & $<.001 \dagger$ \\
\hline Use of DHCA & $12 / 41(29 \%)$ & $10 / 44(23 \%)$ & $5 / 26(19 \%)$ & $67 / 67(100 \%)$ & \\
\hline Total DHCA time (min) & $10.3 \pm 18.2$ & $6.1 \pm 13.1$ & $6.0 \pm 14.2$ & $44.3 \pm 16.0$ & $<.001 \dagger$ \\
\hline Hematocrit $(\%)$ after hemodilution & $28.5 \pm 3.9$ & $25.5 \pm 3.8$ & $28.1 \pm 4.0$ & $28.8 \pm 4.1$ & $<.001 \dagger$ \\
\hline Postoperative length of stay (d) & $10.0 \pm 5.3$ & $6.3 \pm 6.5$ & $3.2 \pm 1.0$ & $15.0 \pm 10.8$ & $<.001 \dagger ; .004 \ddagger$ \\
\hline No. of additional operations before 4-y evaluation & $0.05 \pm 0.2$ & $0.11 \pm 0.4$ & 0 & $2.1 \pm 0.5$ & $<.001 \dagger$ \\
\hline
\end{tabular}

Data presented as mean \pm standard deviation or n (\%). TGA, Transposition of great arteries; TOF, tetralogy of Fallot; VSD, ventricular septal defect; $H L H S$, hypoplastic left heart syndrome; $C P B$, cardiopulmonary bypass; $D H C A$, deep hypothermic circulatory arrest. *TGA versus TOF. $\dagger$ HLHS versus others, TGA versus TOF, TGA versus VSD. $\ddagger$ TGA versus TOF

using the reading and math clusters of the Woodcock-Johnson III, a standardized achievement test for children from 2 years to adulthood. ${ }^{13}$ Fine motor skills were tested using the Wide Range Assessment of Visual Motor Abilities pegboard, a manipulative dexterity test. ${ }^{14}$ All the instruments, except for the GFTA, have an expected mean of 100, with a standard deviation (SD) of 15. The GFTA is scored as a standard percentile ranking according to the number of articulation errors. For all the tests, except the GFTA, moderate impairment was defined as a score of $1 \mathrm{SD}$ and 2 SD less than the expected mean. Severe impairment was defined as a score more than 2 SDs less than the expected mean. In the normative population, approximately $14 \%$ will have a score greater than 1 SD less than the mean and approximately $2.5 \%$ will score more than 2 SDs less than the mean. For the GFTA, impairment was defined as a score of 15 th percentile or less. Not every child was able to complete every test. If a child was judged to be too developmentally impaired to complete the tasks, a score was imputed by assigning them the lowest possible score for the specific test. If the child was unable to complete the task for other reasons, the child was excluded from the analysis for that domain. Additional details concerning the specific tests are provided in the Appendix.

\section{Statistical Analysis}

The APOE genotype was coded for the presence of the $\varepsilon 2$ or $\varepsilon 4$ allele. Subjects with the $\varepsilon 2 \varepsilon 4$ genotype $(\mathrm{n}=14)$ were excluded from the analysis of the APOE genotype effect. The subjects were categorized into 3 groups: $\varepsilon 2(\varepsilon 2 \varepsilon 2$ and $\varepsilon 2 \varepsilon 3), \varepsilon 3(\varepsilon 3 \varepsilon 3)$, and $\varepsilon 4(\varepsilon 3 \varepsilon 4$ and $\varepsilon 4 \varepsilon 4)$. APOE group was coded as a dummy variable, with group $\varepsilon 3$ as the reference. We tested for differences in the variable distributions between HLHS and all other diagnoses and pairwise between TGA and TOF or VSD using a $t$ test or Fisher's exact test. We tested for the association between the diagnosis class and the neurodevelopmental outcomes using the unadjusted and a series of adjusted models. Models incorporating the diagnosis class coded as either HLHS versus all others or for pairwise differences between TGA and TOF or VSD were tested against the same model with the diagnosis class omitted, and the resulting analysis of variance $P$ values are reported. We used 4 adjusted models corresponding to the inclusion of preoperative variables (e.g., ethnicity, gender, socioeconomic class, mother's education, gestational age, birth weight, birth head circumference, intubation, and preoperative length of stay), operative variables (eg, age at surgery, weight at surgery, total CPB time, total DHCA time, use of DHCA, hematocrit, extracorporeal membrane oxygenation/left ventricular assist device, delayed sternal closure, and postoperative length of stay), interval variables (total number of operations, total CPB time, total DHCA time, and use of DHCA during the total interval), and a fully adjusted model combining all variables from the other 3 models.

\section{RESULTS}

Between September 1998 and April 2003, 675 eligible infants underwent cardiac surgery. Of the 675 eligible infants, 23 had died before consent, the parents of 102 declined participation, and $550(81 \%)$ were enrolled. A total of 21 deaths occurred during the initial hospitalization, and an additional 43 patients died before 5 years of age. The study population was $65 \%$ white, $23 \%$ black, and $12 \%$ other ethnic origins; $58 \%$ were male. The largest diagnostic groups were HLHS $(\mathrm{n}=121)$, TOF $(\mathrm{n}=83)$, VSD with or without coarctation $(\mathrm{n}=77)$, and TGA $(\mathrm{n}=45)$. APOE genotyping was completed in $540(98 \%)$ of 550 . The APOE genotype distribution was $\epsilon 2 \epsilon 2$ in $3, \epsilon 2 \epsilon 3$ in $64, \epsilon 2 \epsilon 4$ in $14, \epsilon 3 \epsilon 3$ in $323, \epsilon 3 \epsilon 4$ in 124 , and $\epsilon 4 \epsilon 4$ in 12 and was not different from the Hardy-Weinberg proportions. 


\begin{tabular}{|c|c|c|c|c|c|c|c|c|}
\hline \multirow[b]{2}{*}{ Domain } & \multirow[b]{2}{*}{ All patients } & \multirow[b]{2}{*}{ HLHS } & \multirow[b]{2}{*}{ TGA } & \multirow[b]{2}{*}{ TOF } & \multirow[b]{2}{*}{ VSD } & \multicolumn{3}{|c|}{$P$ values } \\
\hline & & & & & & $\begin{array}{c}\text { HLHS versus } \\
\text { others }\end{array}$ & $\begin{array}{c}\text { TGA versus } \\
\text { TOF }\end{array}$ & $\begin{array}{c}\text { TGA versus } \\
\text { VSD }\end{array}$ \\
\hline Cognition & $100.3 \pm 17.2$ & $94.9 \pm 16.3$ & $105.0 \pm 11.9$ & $103.0 \pm 20.4$ & $102.0 \pm 17.8$ & .001 & .579 & .443 \\
\hline Total language & $102.2 \pm 15.0$ & $99.7 \pm 14.6$ & $103.9 \pm 11.7$ & $103.4 \pm 18.8$ & $104.2 \pm 13.6$ & .078 & .873 & .918 \\
\hline Visual-motor integration & $96.6 \pm 16.0$ & $94.0 \pm 15.7$ & $98.7 \pm 13.5$ & $98.0 \pm 17.7$ & $97.8 \pm 17.3$ & .085 & .825 & .825 \\
\hline Speech production & $49.4 \pm 29.7$ & $50.2 \pm 30.3$ & $46.4 \pm 28.9$ & $51.3 \pm 32.4$ & $48.7 \pm 26.2$ & .765 & .466 & .735 \\
\hline Reading skills & $108.8 \pm 17.0$ & $107.9 \pm 15.8$ & $108.9 \pm 17.6$ & $109.9 \pm 20.9$ & $108.8 \pm 11.3$ & .611 & .812 & .979 \\
\hline Math skills & $100.7 \pm 18.7$ & $96.9 \pm 18.2$ & $105.1 \pm 15.0$ & $100.4 \pm 22.3$ & $104.3 \pm 17.5$ & .037 & .265 & .856 \\
\hline Executive function & $101.8 \pm 13.6$ & $97.7 \pm 14.0$ & $102.7 \pm 10.7$ & $106.7 \pm 14.3$ & $103 \pm 13.1$ & .002 & .163 & .851 \\
\hline Memory & $96.6 \pm 14.8$ & $94.4 \pm 14.2$ & $98.3 \pm 13.4$ & $97.1 \pm 16.8$ & $98.9 \pm 15.1$ & .120 & .709 & .882 \\
\hline Fine motor & $98.4 \pm 15.9$ & $94.1 \pm 15.0$ & $102.3 \pm 11.1$ & $99.7 \pm 17.9$ & $101.1 \pm 19.1$ & .004 & .418 & .761 \\
\hline
\end{tabular}

Data presented as mean \pm standard deviation. Abbreviations as in Table 1.

A total of 486 patients were alive and eligible for the 4year evaluation, which was completed by 381 patients ( $78 \%$ of those eligible). The baseline characteristics have been previously reported for the patients returning for the 4-year evaluation $(\mathrm{n}=381)$, those who did not return $(\mathrm{n}=105)$, and those who died before 4 years old $(\mathrm{n}=64){ }^{7}$ The only significant difference in the baseline characteristics between the returning and nonreturning patients was underrepresentation of blacks in the returning patients ( $21 \%$ versus $29 \%)$.

Of the 381 patients who returned at 4 years, 178 met the entry criteria for the present study: HLHS $(n=67)$, TGA $(\mathrm{n}=41), \operatorname{TOF}(\mathrm{n}=44)$, and VSD $(\mathrm{n}=26)$. No differences
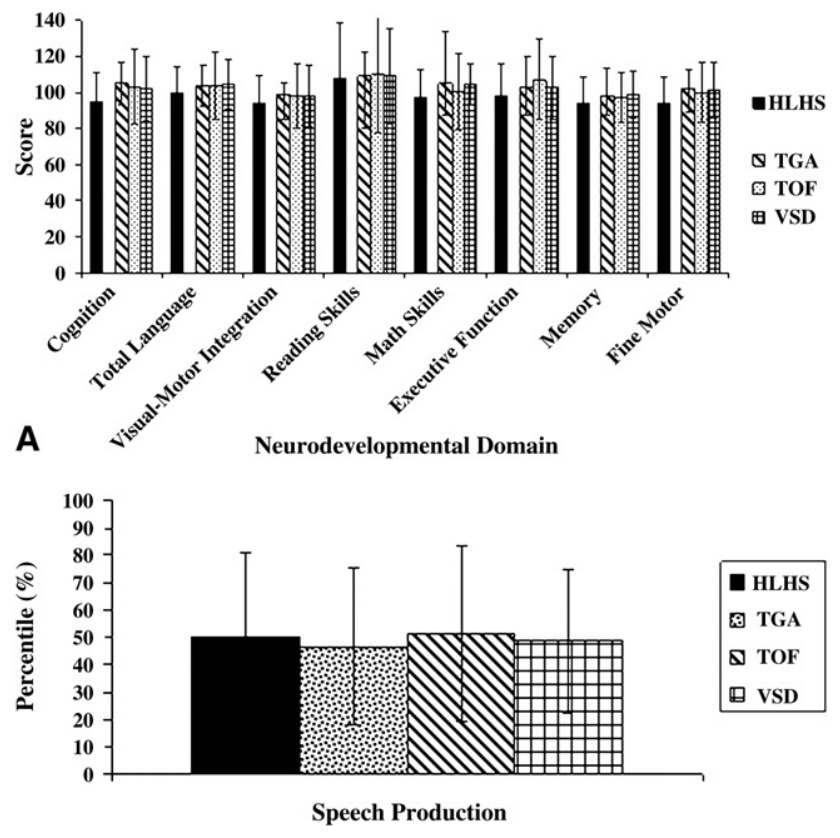

B

Neurodevelopmental Domain

FIGURE 1. A, Mean neurodevelopmental outcome scores for all domains except speech production. All normal scores $100 \pm 15$. B, Mean percentile score for speech production. were found among the diagnostic groups for gender, ethnicity, gestational age, $A P O E$ genotype, maternal education, or socioeconomic status. Significant differences were identified among the diagnostic groups for several baseline and operative management variables (Table 1). The birth weight was lower for the patients with TOF. The use of preoperative mechanical ventilation was more common in patients with TGA and HLHS compared with those with TOF and VSD. The patients with TGA and HLHS underwent their initial operations in the newborn period. In general, the patients with TOF and VSD were older at the initial surgery. The weight at surgery was greater for those with TOF and VSD. The duration of CPB at the first surgery was greater for those with TGA. Both the use and the duration of DHCA were greater for patients with HLHS. The postoperative hospital length of stay was longer for those with HLHS and TGA than for those with TOF or VSD.

All patients with HLHS underwent additional operations with CPB before the 4-year evaluation. Additional operations with $\mathrm{CPB}$ were performed in 4 of the patients with TOF, 2 with TGA, and in none of those with VSD. Also, 55 patients with HLHS underwent additional episodes of DHCA, with a mean total duration of $50 \pm 23$ minutes. DHCA was used in 1 patient with TOF and in none of those with TGA or VSD.

\section{Neurodevelopmental Testing}

The mean unadjusted scores for each domain are listed in Table 2. The mean scores for each diagnostic group were within $1 \mathrm{SD}$ of the expected mean (Figure 1). In general, the mean scores for the patients with HLHS tended to be lower than the other diagnostic groups. Compared with the other patients, those with HLHS had significantly lower scores for cognition, fine motor skills, executive function, and math skills. Using pairwise comparison, no significant differences were found between the patients with TGA and either TOF or VSD for any domain. In general, moderate impairments in at least 1 neurodevelopmental (ND) domain 
TABLE 3. Severity of impairment

\begin{tabular}{|c|c|c|c|c|c|c|c|c|}
\hline \multirow[b]{2}{*}{ Domain } & \multicolumn{4}{|c|}{ Moderate impairment (1-2 SD less than mean) } & \multicolumn{4}{|c|}{ Severe impairment ( $>1$ SD less than mean) } \\
\hline & HLHS & TGA & TOF & VSD & HLHS & TGA & TOF & VSD \\
\hline Cognition & $18 / 67(27)$ & $3 / 41(7)$ & $5 / 44(11)$ & $4 / 26(15)$ & $3 / 67$ (4) & $0 / 41(0)$ & $4 / 44(9)$ & $1 / 26(4)$ \\
\hline Total language & $6 / 66(9)$ & $1 / 41(2)$ & $5 / 44(11)$ & $3 / 25(12)$ & $4 / 66(6)$ & $0 / 41(0)$ & $2 / 44(5)$ & $0 / 25(0)$ \\
\hline Visual-motor integration & $13 / 67(19)$ & $2 / 41(5)$ & $5 / 44(11)$ & $5 / 26(19)$ & $6 / 67(9)$ & $1 / 41(2)$ & $4 / 44(9)$ & $1 / 26(4)$ \\
\hline Speech & $12 / 65(18)$ & $7 / 41(17)$ & $8 / 41(20)$ & $2 / 25(8)$ & & & & \\
\hline Reading & $5 / 66(8)$ & $4 / 41(10)$ & $3 / 43(7)$ & $0 / 23(0)$ & $0 / 66(0)$ & $0 / 41(0)$ & $0 / 43(0)$ & $0 / 23(0)$ \\
\hline Math & $9 / 66(14)$ & $1 / 39(3)$ & $4 / 42(10)$ & $2 / 24(8)$ & $6 / 66(9)$ & $1 / 39(3)$ & $4 / 42(10)$ & $1 / 24(4)$ \\
\hline Executive function & $16 / 66(24)$ & $2 / 41(5)$ & $5 / 40(13)$ & $1 / 25(4)$ & $1 / 66(2)$ & $0 / 41(0)$ & $0 / 40(0)$ & $2 / 25(8)$ \\
\hline Memory & $9 / 64(14)$ & $3 / 41(7)$ & $6 / 40(15)$ & $2 / 24(8)$ & $5 / 64(8)$ & $3 / 41(7)$ & $3 / 40(8)$ & $2 / 24(8)$ \\
\hline Fine motor & $12 / 67(18)$ & $2 / 41(5)$ & $5 / 43(12)$ & $2 / 26(8)$ & $7 / 67(10)$ & $0 / 41(0)$ & $3 / 43(7)$ & $2 / 26(8)$ \\
\hline
\end{tabular}

Data in parentheses are percentages. Abbreviations as in Table 1.

were identified most commonly in patients with HLHS but occurred in all diagnostic groups (Table 3). After correction for the demographic, preoperative, and operative variables, no significant differences were found among the groups for any domain, including the contrast of HLHS to all other groups (Table 4).

\section{DISCUSSION}

ND dysfunction has been increasingly recognized as an important complication of cardiac surgery performed in infancy. We have previously shown that patient-specific factors, such as recognized genetic syndromes and genetic polymorphisms, which modify the brain's response to injury, are significant modulators of the risk of an adverse ND outcome. ${ }^{6,7,15}$ It is known that ND outcomes vary among patients with different cardiac defects. In particular, the outcomes for infants with a functional single ventricle, especially HLHS, had a greater prevalence of adverse ND outcomes compared infants with other types of CHD. ${ }^{4,5,16}$ In general, the reported outcomes for infants with TGA have been superior those with other types of 2-ventricle $\mathrm{CHD}{ }^{3}$ It is not known whether these differences in ND outcomes result from dif-

TABLE 4. Association between HLHS and other diagnoses and neurodevelopmental outcomes using adjusted models

\begin{tabular}{|c|c|c|c|c|}
\hline \multirow[b]{2}{*}{ Domain } & \multicolumn{4}{|c|}{ Analysis of variance $P$ value } \\
\hline & $\begin{array}{c}\text { Preoperative } \\
\text { covariates }\end{array}$ & $\begin{array}{l}\text { Operative } \\
\text { covariates }\end{array}$ & $\begin{array}{c}\text { Interval } \\
\text { covariates }\end{array}$ & $\begin{array}{c}\text { All } \\
\text { covariates }\end{array}$ \\
\hline Cognition & .005 & .108 & .543 & .950 \\
\hline Total language & .204 & .634 & .435 & .942 \\
\hline Visual-motor integration & .054 & .764 & .749 & .873 \\
\hline Speech production & .889 & .038 & .592 & .495 \\
\hline Reading skills & .905 & .504 & .278 & .502 \\
\hline Math skills & .070 & .936 & .197 & .355 \\
\hline Executive function & .014 & .269 & .026 & .256 \\
\hline Memory & .139 & .485 & .439 & .705 \\
\hline Fine motor skills & .038 & .529 & .495 & .971 \\
\hline
\end{tabular}

ferences in central nervous system development related to the type of CHD, differences in the physiologic effects of the specific defect, or diagnosis-specific differences in management. In addition, the association of genetic anomalies that include both CHD and ND dysfunction varies according to the type of CHD. Microdeletions of the 22nd chromosome are more commonly identified in patients with TOF compared with TGA. ${ }^{17,18}$

In the present study, we evaluated outcomes for $8 \mathrm{ND}$ domains in preschool children after surgery in infancy for TGA, VSD, TOF, and HLHS. We specifically excluded patients with known or suspected genetic anomalies. The mean unadjusted outcomes for all domains in all 4 diagnostic groups were within the normal range $( \pm S D)$ for the general population. However, the number of children with moderate to severe impairment in at least $1 \mathrm{ND}$ domain was greater than expected for the general population for all diagnostic groups. The differences among the diagnostic groups were less than might be predicted from previous research. In general, the mean scores for the patients with HLHS tended to be lower than those for the other diagnostic groups. ${ }^{16,19}$ These differences were significant for 4 of the 8 developmental domains evaluated. Unlike in previous studies, no significant differences were found among the patients with TGA, TOF, or VSD for unadjusted outcomes for any domain. This finding might have been secondary to careful evaluation for genetic syndromes and exclusion of patients with known or suspected genetic anomalies. ${ }^{17}$

Significant differences were found among the diagnostic groups for several factors that might adversely affect ND outcomes, including preoperative mechanical ventilation and postoperative length of stay. As would be expected, at least moderately strong correlations were found between the cardiac diagnosis and several operative management variables. In addition, unlike the other patient groups, the patients with HLHS undergo multiple operations and remain hypoxemic until completion of the Fontan procedure, usually between 2 and 3 years of age. These differences in 
management and prolonged exposure to hypoxemia were significantly linked to the specific cardiac diagnosis. After correction for preoperative and operative factors, we could no longer identify significant differences among the diagnostic groups for any ND domain.

We previously evaluated the outcomes for our patients with HLHS at 1 year of age. ${ }^{5}$ In that report, we noted that the mean scores for the Bayley Scales of Infant Development-II were lower than those of the population norms, and many children had scores more than 2 SD less than the mean signifying severe impairment. We included children with genetic anomalies in that analysis and presence of such an anomaly was a significant predictor of worse performance. The present study included only children with no evidence of genetic syndromes, which likely explains the better performance for the HLHS subgroup.

The present study had limitations. The management of CPB was not standardized for the present study. By the nature of their defect, children with HLHS experience prolonged exposure to hypoxemia compared with children with 2-ventricle CHD. However, we did not assess the severity or duration of the hypoxemia. Although we evaluated multiple ND domains, these might have provided an incomplete assessment of the potential developmental disabilities, especially behavioral problems. In particular, the young age of the children makes an assessment of academic abilities difficult.

\section{CONCLUSIONS}

The mean scores for the ND domains tested were in the normal range for preschool children with no recognized genetic syndromes after surgery for VSD, TOF, TGA, or HLHS. The number of children with moderate to severe impairments in at least 1 domain was increased compared with the general population for all diagnostic groups. The unadjusted ND outcomes for those with HLHS were lower for cognition, fine motor skills, executive function, and math skills compared with the other patients. After correction for the demographic, preoperative, and operative variables, no significant differences were seen among the 4 groups for any domain. The specific cardiac diagnosis determined a large portion of the variation in these covariates. Therefore, although HLHS did predict for poorer outcomes in some domains, it did not add predictive power to the other factors considered. From our results, it was not possible to determine whether the cardiac diagnosis is causal in its prediction of ND outcomes or related secondary to covariates. Unlike previous studies, we found no significant differences in ND outcomes among the patients with TGA, TOF, or VSD. This likely resulted from our exclusion of patients with suspected or confirmed genetic anomalies. This finding highlights the importance of genetic factors as a determinant of ND outcomes in the CHD population. Future studies of ND outcomes or neuroprotective strategies in this popu- lation should include formal genetic evaluation and stratification by the presence of genetic anomalies.

\section{References}

1. Shillingford AJ, Glanzman MM, Ittenbach RF, Clancy RR, Gaynor JW, Wernovsky G. Inattention, hyperactivity and school performance in a population of school age children with complex congenital heart disease. Pediatrics. 2008; 121:e759-67.

2. Wernovsky G, Shillingford AJ, Gaynor JW. Central nervous system outcomes in children with complex congenital heart disease. Curr Opin Cardiol. 2005;20 94-9.

3. Bellinger D, Wypij D, du Plessis A, Rappaport L, Riviello J, Jonas R, et al. Developmental and neurologic effects of alpha-stat versus $\mathrm{pH}$-stat strategies for deep hypothermic cardiopulmonary bypass in infants. J Thorac Cardiovasc Surg. 2001;121:374-83.

4. Forbess JM, Visconti KJ, Hancock-Friesen C, Howe RC, Bellinger DC, Jonas RA. Neurodevelopmental outcome after congenital heart surgery: Results from an institutional registry. Circulation. 2002;106(12 Suppl. 1):I95-102.

5. Tabbutt S, Nord AS, Jarvik GP, Bernbaum J, Wernovsky G, Gerdes M, et al. Neurodevelopmental outcomes after staged palliation for hypoplastic left heart syndrome. Pediatrics. 2008;121:476-83.

6. Gaynor JW, Gerdes M, Zackai EH, Bernbaum J, Wernovsky G, Clancy RR, et al. Apolipoprotein E genotype and neurodevelopmental sequelae of infant cardiac surgery. J Thorac Cardiovasc Surg. 2003;126:1736-45.

7. Gaynor JW, Nord A, Wernovsky G, Bernbaum J, Solot C, Burnham N, et al. Apolipoprotein E genotype modifies the risk of behavior problems in preschool children following neonatal and infant cardiac surgery. Pediatrics. 2009;124:241-50.

8. Wechsler D. Wechsler Preschool and Primary Scale of Intelligence. 3rd ed. San Antonio, TX: Harcourt Assessment Co; 2002.

9. Zimmermanr I, Steiner VP, Evatt R. Preschool Language Scale-IV. 4th ed. San Antonio, TX: Harcourt Assessment; 2002.

10. Korkman M, Kirk U, Kemp S. NEPSY. 2nd ed. San Antonio, TX: PsychCorp/ Harcourt Assessment; 2007.

11. Beery K. The VMI: Developmental test of visual motor integration-Administration, scoring and testing manual. Cleveland, OH: Modern Circulation Press; 1989.

12. Goldman F. Golden Fristoe Test of Articulation-2. Circle Pines, MN; 2000

13. Mather N, Woodcock R. Woodcock Johnson Achievement Battery III Manuel. Third ed. Illinois: Riverside Publishing; 2001.

14. Adams, D. Wide range assessment of visual motor abilities. Wilmington, DE 1995

15. Gaynor JW, Wernovsky G, Jarvik GP, Bernbaum J, Gerdes M, Zackai E, et al. Patient characteristics are important determinants of neurodevelopmental outcome at one year of age after neonatal and infant cardiac surgery. J Thorac Cardiovasc Surg. 2007;133:1344-53.

16. Mahle WT, Wernovsky G. Neurodevelopmental outcomes in hypoplastic left heart syndrome. Semin Thorac Cardiovasc Surg Pediatr Card Surg Annu. 2004; 7:39-47.

17. Zeltser I, Jarvik GP, Bernbaum J, Wernovsky G, Nord AS, Gerdes M, et al. Genetic factors are important determinants of neurodevelopmental outcome after repair of tetralogy of Fallot. J Thorac Cardiovasc Surg. 2008;135:91-7.

18. Gerdes M, Solot C, Wang PP, Moss E, LaRossa D, Randall P, et al. Cognitive and behavior profile of preschool children with chromosome 22q11.2 deletion. Am J Med Genet. 1999;85:127-33.

19. Goldberg CS, Schwartz EM, Brunberg JA, Mosca RS, Bove EL, Schork MA, et al. Neurodevelopmental outcome of patients after the Fontan operation: A comparison between children with hypoplastic left heart syndrome and other functional single ventricle lesions. J Pediatr. 2000;137:646-52.

\section{APPENDIX}

The Wechsler Preschool and Primary Scale of Intelligence, 3rd edition (WPPSI-III) is a standardized test of intelligence for children 3.5 to 7 years old. ${ }^{8}$ It is commonly used in both clinical settings and research. It takes approximately 45 minutes to administer and yields 3 summary scores and 12 subtest scores. The scores include the fullscale IQ, verbal IQ, and performance IQ, with a mean score 
of 100 and standard deviation of 15 points. It covers a wide range of cognitive tasks. A large body of data is available to explain the meaning of the test findings. The WPPSIrevised has been proved to have moderate to strong reliability (the coefficient for verbal, performance, processing speed, full and general language was 0.92, 0.87, 0.93, 0.92 , and 0.90 , respectively) and validity (correlation with other cognitive tests in the positive and significant range of 0.74-0.90) in a variety of studies.

The Preschool Language Test- 4 is a general test of early language skills. ${ }^{9}$ It provides a measure of language comprehension and expressive communication. The standard scores are derived according to age and performance. The total language score (mean 100, with a standard deviation of 15) is derived according to performance on the receptive and expressive sections.

The Developmental Test of Visual Motor Integration (VMI) is a copying task used to assess a child's fine motor and visual motor coordination skills. ${ }^{11}$ It takes 10 minutes to complete and yields standard scores with a mean of 100 and standard deviation of 15 . It is a well-recognized test, and the new edition was published in 1989. Handedness is also noted on the VMI. The interrater reliability had a median of 0.93. Generally, researchers have found the VMI to be a valuable predictor when used in combination with other measures. The positive correlation with other tests of visual skills and motor skills was documented in the manual as ranging from 0.72 to 0.76 .

The Goldman Fristoe Test of Articulation 2 (GFTA) is a systematic measure of assessing an individual's articulation of the consonant sounds of Standard American English. Scores are represented in percentiles according to age and performance. ${ }^{12}$ The time for testing is 10 to 15 minutes.

The Woodcock-Johnson III is a standardized achievement test for children 2 years to adulthood. It has recently been normed and revised. ${ }^{13}$ It has subtests that measure achievement and cognitive skills. For the purpose of the present study, only the reading and math clusters were used. These subtests take about 10 minutes each and measure a preschooler's skills in these areas. The norming procedures of the Woodcock-Johnson III were excellent and reflected the most recent census data. The data on reliability ranged from 0.80 to 0.87 for individual tests. This is one of the few achievement tests that have been normed for preschoolers.

The Neuro-PSYchology (NEPSY) statue test is a developmental neuropsychological assessment tool published in 1998. Korkman and colleagues. ${ }^{10}$ The NEPSY Statue subtest assesses inhibition and motor persistence. The NEPSY subtests yield scale scores with a mean of 10 and a standard deviation of 3 . The reliability ranges from 0.50 to 0.81 . The validity studies indicated a low correlation between the attention/executive function subtests and tests of general intelligence. The use of the NEPSY with clinical populations of children diagnosed with attention deficit hyperactivity disorder has shown that identified children score significantly poorer on the tests of attention.

The Wide Range Assessment of Visual Motor Abilities pegboard is a manipulative dexterity test. ${ }^{14}$ The child inserts as many pegs as possible within 90 seconds using a nearly square pegboard. The pegboard is "waffled" to add to its fine motor demands, as well as to increase its esthetic appeal. The test is completed first with the dominant hand and then with the nondominant hand. This test was chosen from the collection of pegboard tasks (e.g., the Purdue pegboard and grooved pegboard), because it is the only one designed and standardized for a 4-year-old population. The scores are provided as standard scores and percentiles, which were published in 1995 . The reliability and validity for the proposed age group are strong.

\section{Discussion}

Dr Ivan M. Rebeyka (Edmonton, Alberta, Canada). Neurodevelopmental outcome has now eclipsed mortality as the new yardstick or scorecard by which the results of congenital heart surgery are presently evaluated and compared. Ten years ago, the referring cardiologist seemed quite satisfied when the patient survived to discharge; now, they expect them to be future candidates for membership in the Mensa Society.

Dr. Gaynor and colleagues at the Children's Hospital of Philadelphia have been leaders in this area of investigation and have made many contributions, including the demonstration that much of the variance seen in developmental outcomes after congenital cardiac surgery is secondary to unmodifiable patient characteristics related to a genetic predisposition and that the surgeon no longer should feel guilty about every suboptimal outcome, because it is not always their fault.

This report describes the neurodevelopmental outcomes of a cohort of 178 patients who had undergone repair before 6 months of age and then underwent a detailed neurodevelopmental assessment at 4 years of age. This type of study clearly represents a tremendous amount of work and the results warrant careful study.

The results at first glance seem almost too good to be true, with average unadjusted cognition scores ranging from 94 for those with hypoplastic left heart syndrome to 105 for those with transposition. However, Dr Gaynor has also indicated that the study patients had been selected by excluding those with either a definite or suspected genetic abnormality.

Thus, my first question relates to the total number of children not included in the analysis. If I read the manuscript correctly, 381 patients had undergone the 4-year evaluation, but the results for only 178 patients were included in the analysis, suggesting that more than one half of the patients were excluded. Thus, although a definite chromosomal abnormality such as trisomy 18 or 21 is obvious, the other classifications of a suspected genetic abnormality must have been more subjective. Therefore, I would ask by what criteria the genetic dysmorphologist excluded those patients with a suspected genetic abnormality, because there really seems to be a disproportionately high number of children with genetic syndromes among these 4 diagnostic groups. 
Dr Gaynor. As I stated, all these patients underwent a detailed examination by the dysmorphologist. Also, many of the patients, particularly in this group-most of the ones who had genetic syndromes in this group were in the tetralogy group. Most of those had confirmed microdeletions of the 22 nd chromosome. A variety of other defects were present in terms of translocations and other genetic abnormalities we found on chromosomal testing and a variety of other syndromes-Charge syndrome, Pierre Robin syndrome, Kabuki syndrome - that were not picked up at birth.

When we set up this cohort, we specifically tried to exclude patients with any genetic syndrome; however, as we have demonstrated, it can be very difficult to recognize dysmorphic features in the newborn period because of the infant's features. Thus, at 1 year of age, this was determined by the examination findings, the presence of dysmorphic features, and the genetic testing recommended by our geneticist.

Dr Rebeyka. It just seems high that well over one half had some type of genetic syndrome.

Dr Gaynor. Well, this was not all half. Again, because we specifically wanted to compare specific cardiac diagnoses, we have about-if you consider the whole cohort, we had about $20 \%$ or $20 \%$ to $25 \%$ who have a definite or suspected syndrome. We excluded many of the cardiac diagnoses; for example, 2 children with an aortopulmonary window and a small number with every other defect one can imagine, including anomalous left coronary, anomalous pulmonary veins, truncus arteriosus, interrupted aortic arch. We excluded those patients because the subgroups in any particular group were not enough to allow an analysis to study the cardiac defect and the cardiac diagnosis, which was the purpose of this study.

Dr Rebeyka. My second question relates to the use of the mean scores as a potentially misleading indicator of neurodevelopmental outcome. One would be hard pressed not to be pleased with average scores of 95 for those with hypoplastic left heart syndrome, 104 for tetralogy, and a similar value of 104 for VSD, as your re- sults indicate. However, considering the number of patients having either a moderate or severe degree of impairment, the outcomes are not quite so optimistic, as you pointed out, with $35 \%$ of those with hypoplastic left heart syndrome having either moderate or severe impairment, and corresponding percentages for those with tetralogy and VSD of $29 \%$ and $23 \%$. Although it might be understandable to observe this degree of impairment in the hypoplastic group, it seems disturbing that $29 \%$ of those with tetralogy and $23 \%$ of those with VSD had either a moderate or severe degree of impairment. Can you comment on that?

Dr Gaynor. Particularly with those with VSD, we were surprised at how poorly they did. But, again, you have to remember this is, particularly for those with VSD, this is a subgroup. A tetralogy of Fallot repair is usually done at 2 to 3 months of age at our institution. These VSD cases were almost always selected because they were children who had severe heart failure and failure to thrive and, usually, suboptimal weight gain. If you looked at the weights, the weights of the VSD patients were significantly less than the weights of the tetralogy patients. I think, at least for the VSD cases, I can understand that these children had had failure to thrive and poor growth in the early postnatal period, which is critical for brain development. So, I think this is not all patients with VSD, this is patients with VSD who came to surgery very early in life, usually at around 2 months of age, and almost always because of failure to thrive.

In terms of those with tetralogy, I am not sure why they had that degree of impairment.

Dr Rebeyka. My last question is a real easy one. Your series of 178 patients includes 67 with hypoplastic left heart syndrome and 25 with VSD. In Philadelphia, do you really perform 2.5 times as many hypoplastic repairs as VSD repairs?

Dr Gaynor. Again, this is all patients younger than 6 months of age; a lot of our VSD patients are older than 6 months of age. So again, this is by specific entry criteria for this study. 